\title{
PERMUTATIONS BY NUMBER OF RISES AND SUCCESSIONS
}

\author{
D. P. ROSELLE ${ }^{1}$
}

1. Introduction. Permutations with restricted positions are treated at length in Riordan's book [4]. Two of the problems considered are the "problème des rencontres" and the enumeration of permutations by number of rises in the permutation. The former asks for the number of permutations

$$
\left(\begin{array}{llll}
1 & 2 & \cdots & n \\
l_{1} & l_{2} & \cdots & l_{n}
\end{array}\right)
$$

such that $l_{j} \neq j(j=1(1) n)$ and the latter asks for the number of permutations for which there are a fixed number of $j$ such that $l_{j}<l_{j+1}$.

Two notational conventions will prove useful in what follows. First, it shall be convenient to write the permutation (1.1) as the one line array $\left(l_{1}, l_{2}, \cdots, l_{n}\right)$ and, secondly, when enumerating permutations by number of rises, we shall attach an initial rise. Thus, for example, the permutations

$$
231, \quad 1432
$$

each have two rises. Notice that the first of these is admissible as a recontres permutation, whereas the second is not admissible.

Let $D_{n}$ denote the number of rencontres permutations. Then $[4$, p. 59$]$

$$
D_{n}=n D_{n-1}+(-1)^{n}=\sum_{j=0}^{n}(-1)^{j}(n ! / j !) .
$$

Also, if $A(n, k)$ denotes the number of permutations (1.1) with exactly $k$ rises, then $A(1, k)=\delta_{1 k}$ (Kronecker delta) and

$$
A(n, k)=k A(n-1, k)+(n+1-k) A(n-1, k-1) .
$$

It follows from (1.3) that $A(n, k)$ is the Eulerian number [1], [2] given by the formula

$$
A(n, k)=\sum_{j=0}^{k}(-1)^{j}\left(\begin{array}{c}
n+1 \\
j
\end{array}\right)(k-j)^{n} .
$$

Received by the editors July 18, 1966 and, in revised form, October 3, 1966.

${ }^{1}$ Supported by the General Research Board of the University of Maryland. 
Next, we define a succession in a permutation (1.1) as an element pair $\left(l_{j}, l_{j+1}\right)$ with $l_{j+1}=l_{j}+1$. Permutations without successions are enumerated in $[5$, p. 103] and the enumerator by number of successions in [6]

$$
S_{n}(x)=D_{n}(x)+(1-x) D_{n-1}(x),
$$

with

$$
D_{n}(x)=(D+x)^{n}, \quad D^{j} \equiv D_{j},
$$

the rencontres polynomial.

Here we are concerned with enumerating permutations both by number of rises and number of successions. The author is indebted to the referee for many helpful suggestions and for pointing out references [5] and [6].

2. Reduction of the problem. Let $P(n, r, s)$ denote the number of permutations (1.1) which have exactly $r$ rises and $s$ successions. We prove that

$$
P(n, r, s)=\left(\begin{array}{c}
n-1 \\
s
\end{array}\right) P(n-s, r-s, 0) .
$$

Fix $n, r$, and $s$ and let $P$ and $Q$ denote the sets of permutations enumerated by $P(n, r, s)$ and $P(n-s, r-s, 0)$, respectively.

Given $\pi \in P$, we delete the $s$ elements which occur as the second element of a succession in $\pi$. This defines a mapping of $P$ onto the set $S$ of sequences.

$$
\rho=\left(j_{1}, j_{2}, \cdots, j_{n-s}\right)
$$

containing 1, having $r-s$ rises, and such that, if the elements are ordered,

$$
1=j_{k_{1}}<j_{k_{2}}<\cdots<j_{k_{n-\text { s }}} \leqq n,
$$

then there is no pair

$$
\left(j_{m}, j_{m+1}\right)=\left(j_{k_{t}}, j_{k_{t+1}}\right) \quad(1 \leqq m, t \leqq n-1-s) .
$$

For if $\rho$ contained a pair $\left(j_{m}, j_{m+1}\right)$ satisfying (2.4) then, replacing the elements deleted from $\pi$, we see that the succession $\left(j_{m+1}-1, j_{m+1}\right)$ occurs in $\pi$. This contradicts the occurrence of $j_{m+1}$ in $\rho$ or, if we prefer, shows that $\pi$ has at least $s+1$ increases.

The inverse of the mapping defined above can be described explicitly. For if $\rho \in S$ and $t_{1}<\cdots<t_{s}$ are the elements of $\{2,3, \cdots, n\}$ not occurring in $\rho$, we define the permutation $\pi$ inductively by first 
inserting $t_{1}$ immediately to the right of $t_{1}-1$ in $\rho$ and, at the $i$ th stage, $t_{i}$ immediately to the right of $t_{i}-1$. It is easy to verify that the permutation $\pi$ constructed in this way corresponds, under the correspondence of the last paragraph, to the sequence $\rho$. Thus

$$
|S|=|P| \text {. }
$$

On the other hand, given $\rho \in S$, ordered according to (2.3), we define a permutation $\sigma$ of $\{1,2, \cdots, n-s\}$ by replacing $j_{k_{t}}$ by $t$ in $\rho$. It is clear that $\sigma$ has rises in exactly the same positions as $\rho$, hence a total of $r-s$ rises. Moreover, (2.4) assures us that $\sigma$ has no successions. Thus $\sigma \in Q$. That the mapping from $S$ to $Q$ defined in this way is on to is immediate when we notice that $Q \subseteq S$.

However, the mapping from $S$ to $Q$ is many-to-one. Indeed, given $\sigma \in Q$, we select $n-1-s$ elements

$$
j_{k_{2}}<j_{k_{3}}<\cdots<j_{k_{n-\text { s }}}
$$

from the set $\{2,3, \cdots, n\}$ and replace $t$ by $j_{k_{t}}$ in $\sigma$. The resulting sequence $\rho$ has rises in the same positions as $\sigma$ and, since $\sigma$ has no successions, $\rho$ has no pair satisfying (2.4). We easily verify that this sequence $\rho$ corresponds to $\sigma$ and that any sequence which corresponds to $\sigma$ can be constructed in this manner. Therefore, since the elements (2.6) can be chosen in

$$
\left(\begin{array}{c}
n-1 \\
n-1-s
\end{array}\right)=\left(\begin{array}{c}
n-1 \\
s
\end{array}\right)
$$

ways, we get

$$
|S|=\left(\begin{array}{c}
n-1 \\
s
\end{array}\right)|Q|
$$

which, together with (2.5), implies (2.1).

\begin{tabular}{cccc}
\multicolumn{2}{c}{$\sigma=132$} & \multicolumn{2}{c}{$\sigma=213$} \\
$\rho$ & $\pi$ & $\rho$ & $\pi$ \\
132 & 13452 & 213 & 21345 \\
142 & 14523 & 214 & 23145 \\
152 & 15234 & 215 & 23415 \\
143 & 12453 & 314 & 31245 \\
153 & 12534 & 315 & 34125 \\
154 & 12354, & 415 & 41235.
\end{tabular}


We easily verify that $P(3,2,0)=2$. Thus by $(2.1)$

$$
P(s+3, s+2, s)=2 \cdot\left(\begin{array}{c}
s+2 \\
2
\end{array}\right)=(s+1)(s+2)
$$

and, for $s=2$, the preceding table illustrates the correspondences given above.

3. The numbers $P(n, r)$. In view of (2.1) it will suffice to determine the numbers $P(n, r)=P(n, r, 0)$.

Noting that the number $s$ of successions in a permutation satisfies the inequalities $0 \leqq s<r$, it follows from the combinatorial interpretation of the Eulerian numbers that

$$
\sum_{s=0}^{r-1} P(n, r, s)=A(n, r) .
$$

But, using (2.1), this entails

$$
\sum_{s=0}^{r-1}\left(\begin{array}{c}
n-1 \\
s
\end{array}\right) P(n-s, r-s)=A(n, r),
$$

which is equivalent to

$$
\sum_{s=0}^{r-1}(-1)^{s}\left(\begin{array}{c}
n-1 \\
s
\end{array}\right) A(n-s, r-s)=P(n, r) .
$$

We remark that (3.3) can be proved directly using the principle of inclusion and exclusion [4, p. 50] and that, together with (1.4), it implies an explicit formula for $P(n, r)$. Notice also that

$$
P(n, r)=0 \quad(r \geqq n)
$$

follows from (3.3).

Next, if we define the polynomials

$$
\begin{aligned}
& P_{n}(t)=\sum_{r=1}^{n-1} P(n, r) t^{r} \\
& A_{n}(t)=\sum_{r=1}^{n} A(n, r) t^{r}
\end{aligned}
$$

then (3.3) implies

$$
P_{n}(t)=A(t)(A(t)-t)^{n-1}, \quad A^{j}(t) \equiv A_{j}(t) .
$$

It follows that 


$$
\begin{aligned}
P_{n}(t) & =(A(t)-t+t)(A(t)-t)^{n-1} \\
& =(A(t)-t)^{n}+t(A(t)-t)^{n-1} .
\end{aligned}
$$

Thus if we put

$$
P_{n}^{*}(t)=(A(t)-t)^{n}=\sum_{r=1}^{n-1} P^{*}(n, r) t^{r}
$$

we get

$$
P_{n}(t)=P_{n}^{*}(t)+t P_{n-1}^{*}(t) .
$$

We shall see that the polynomial $P_{n}^{*}(t)$ is more convenient than $P_{n}(t)$.

4. Recurrences. Writing

$$
\begin{aligned}
& P^{*}(t, u)=\sum_{n=0}^{\infty} P_{n}^{*}(t) \frac{u^{n}}{n !}, \\
& A(t, u)=\sum_{n=0}^{\infty} A_{n}(t) \frac{u^{n}}{n !}=\frac{1-t}{1-t \exp [u(1-t)]},
\end{aligned}
$$

we see from 3.7 that

$$
P^{*}(t, u)=e^{-t u} A(t, u)
$$

and, with subscripts denoting partial derivatives,

$$
(1-t u) A_{u}(t, u)=t A(t, u)+t(1-t) A_{t}(t, u) .
$$

Consequently

$$
(1-t u) P_{u}^{*}(t, u)=t u P^{*}(t, u)+t(1-t) P_{t}^{*}(t, u)
$$

which entails, with $D=d / d t$,

$$
P_{n+1}^{*}(t)=n t\left(P_{n}^{*}(t)+P_{n-1}^{*}(t)\right)+t(1-t) D P_{n}^{*}(t) .
$$

Note that, using (3.7), (3.8), and (4.5), we obtain the recurrence

$$
\begin{aligned}
P^{*}(n+1, r)= & r P^{*}(n, r)+(n+1-r) P^{*}(n, r-1) \\
& +n P^{*}(n-1, r-1)
\end{aligned}
$$

and, since

$$
P(n, r)=P^{*}(n, r)+P^{*}(n-1, r-1),
$$

we have also

$$
\begin{aligned}
P(n+1, r)= & r P(n, r)+(n+1-r) P(n, r) \\
& +(n-1) P(n-1, r-1) .
\end{aligned}
$$


The following tables of $P^{*}(n, r)$ and $P(n, r)(1 \leqq r<n \leqq 7)$ are easily computed using (4.6) and (4.7).

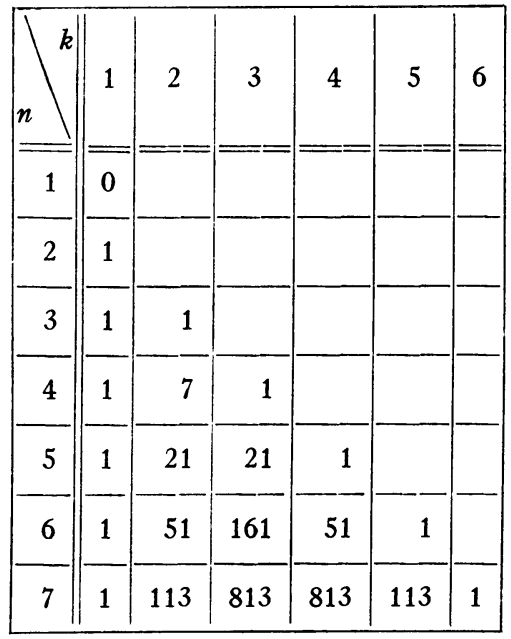

\begin{tabular}{|c|c|c|c|c|c|c|}
\hline${ }_{n}^{k}$ & 1 & 2 & 3 & 4 & 5 & 6 \\
\hline 1 & 1 & & & & & \\
\hline 2 & 1 & & & & & \\
\hline 3 & 1 & 2 & & & & \\
\hline 4 & 1 & 8 & 2 & & & \\
\hline 5 & 1 & 22 & 28 & 2 & & \\
\hline 6 & 1 & 52 & 182 & 72 & 2 & \\
\hline 7 & 1 & 114 & 864 & 974 & 164 & 2 \\
\hline
\end{tabular}

5. The polynomial $P_{n}^{*}(t)$. We remark that the combinatorial interpretation of $P(n, r)$ furnishes a similar interpretation of $P^{*}(n, r)$. Indeed, it follows from (4.7) that $P^{*}(n, r)$ is the number of permutations (1.1) with $r$ rises, no successions, and $l_{1}>1$.

In the next place we have, using (4.2) and (4.3),

$$
P^{*}(t, u)=P^{*}\left(t^{-1}, t u\right),
$$

which is equivalent to either of

$$
\begin{aligned}
P_{n}^{*}(t) & =t^{n} P_{n}^{*}\left(t^{-1}\right), \\
P^{*}(n, r) & =P^{*}(n, n-r) .
\end{aligned}
$$

Notice that, using (3.7) and the familiar symmetry property of the Eulerian numbers, we have

$$
\begin{aligned}
t^{n+1} A_{n}\left(t^{-1}\right) & =A_{n}(t)=t^{n+1}\left(P^{*}\left(t^{-1}\right)+t^{-1}\right)^{n} \\
& =t\left(t P^{*}\left(t^{-1}\right)+1\right)^{n},
\end{aligned}
$$

which, in view of (5.2), implies, for $n \geqq 1$,

$$
A_{n}(t)=t\left(P^{*}(t)+1\right)^{n}, \quad P^{* j}(t) \equiv P_{j}^{*}(t) .
$$

In exactly the same way we find that

$$
t P_{n}^{*}(t)=(A(t)-1)^{n}+(-1)^{n}(t-1) .
$$


The relations (5.4) and (5.5) are more convenient for calculation than (3.7).

Next we define the polynomial

$$
P_{n}^{*}(a, t)=\left(a+P^{*}(t)\right)^{n}, \quad P^{* j}(t) \equiv P_{j}^{*}(t),
$$

so that

$$
\begin{aligned}
t P_{n}^{*}(1, t) & =A_{n}(t)=P_{n}^{*}(t, t), \\
P_{n}^{*}(0, t) & =P_{n}^{*}(t) .
\end{aligned}
$$

Writing $F(x, a, t)=\sum P_{n}^{*}(a, t) x^{n} / n$ !, we find using (4.2) and (4.3) that

$$
F(x, a, t)=\frac{(1-t) \exp [(a-1) x]}{\exp [x(t-1)]-t},
$$

which is equivalent to

$$
P_{n}^{*}(a, t)=(t-1)^{n} H_{n}\left(\frac{a-1}{t-1} \mid t\right),
$$

with $H_{n}(u \mid \lambda)$ defined [1] by

$$
\frac{(1-\lambda) e^{x u}}{e^{x}-\lambda}=\sum_{n=0}^{\infty} H_{n}(u \mid \lambda) \frac{x^{n}}{n !} .
$$

Notice also that (5.8) implies the recurrence

$$
\left[P^{*}(a, t)+(t-1)\right]^{n}=P_{n}^{*}(a, t)+(1-t)(a-1)^{n}
$$

6. Special cases. Taking $t=-1$ in (5.9), we get

which implies

$$
\sum_{n=0}^{\infty} P_{n}^{*}(a,-1) \frac{x^{n}}{n !}=\frac{2 e^{(a+1) x}}{e^{2 x}+1},
$$

$$
P_{n}^{*}(a,-1)=2^{n} E_{n}(a),
$$

with $E_{n}(a)$ the Euler polynomial. In particular,

$$
P_{n}^{*}(-1)=2^{n} E_{n}(0)=E_{n},
$$

with $E_{n}$ the Euler number. For properties of Euler numbers and polynomials, the reader is referred to [3].

Next it follows from (4.5) that

$$
\begin{aligned}
P_{n+1}^{*}(1) & =n P_{n}^{*}(1)+n P_{n-1}^{*}(1) \\
& =(n+1) P_{n}^{*}(1)+(-1)^{n+1}
\end{aligned}
$$


Comparing this with (1.2) we see that

$$
P_{n}^{*}(1)=D_{n},
$$

the rencontres number. Thus, using (6.2), we obtain

$$
P_{n}^{*}(a, 1)=D_{n}(a),
$$

the rencontres polynomial.

It is clear from the combinatorial meaning of $P(n, r, s)$ that the polynomial

$$
P_{n}(x, t)=\sum_{r=1}^{n} \sum_{s=0}^{r-1} P(n, r, s) x^{8} t^{r}
$$

satisfies

$$
\begin{aligned}
& P_{n}(x, 1)=S_{n}(x), \\
& P_{n}(1, t)=R_{n}(t),
\end{aligned}
$$

where $S_{n}(x)$ and $R_{n}(t)$ are the enumerators for permutations by number of successions and rises, respectively. Also it follows from (2.1) and (3.8) that

$$
\begin{aligned}
P_{n}(x, t) & =\sum_{s=0}^{n-1}\left(\begin{array}{c}
n-1 \\
s
\end{array}\right) x^{s} t^{8} P_{n-s}(t) \\
& =\sum_{s=0}^{n-1}\left(\begin{array}{c}
n-1 \\
s
\end{array}\right) x^{s} t^{s}\left\{P_{n-s}^{*}(t)+t P_{n-s-1}^{*}(t)\right\} \\
& =P^{*}(t)\left(P^{*}(t)+x t\right)^{n-1}+t\left(P^{*}(t)+x t\right)^{n-1}
\end{aligned}
$$

which is the same as

$$
P_{n}(x, t)=\left(P^{*}(t)+x t\right)^{n}+t(1-x)\left(P^{*}(t)+x t\right)^{n-1},
$$

or, using (5.6),

$$
P_{n}(x, t)=P_{n}^{*}(x t, t)+t(1-x) P_{n-1}^{*}(x t, t) .
$$

Consequently it follows from (6.4) and (6.6) that

$$
S_{n}(x)=D_{n}(x)+(1-x) D_{n-1}(x)
$$

and from (5.7) that

$$
R_{n}(t)=A_{n}(t)
$$

are the enumerators for permutations by number of successions and rises.

We remark that the generating function for the polynomial defined by (6.5) can be found by combining (5.9) and (6.9). 


\section{REFERENCES}

1. L. Carlitz, Eulerian numbers and polynomials, Math. Mag. 30 (1958), 203-214.

2. G. Frobenius, Über die Bernoullischen Zahlen und die Eulerschen Polynome, S.-B. Preuss. Akad. Wiss. (1910), 809-847.

3. N. Nielsen, Traité élémentaire des nombres de Bernoulli, Paris, 1923.

4. J. Riordan, An introduction to combinatorial analysis, Wiley, New York, 1958.

5. H. Tietze, Über gewisse Unordnungen von Permutationen, S.-B. Math.-Natur. Abt. Bayer. Akad. Wiss. (1943), 281-293.

6. W. A. Whitworth, Choice and chance, Stechert, New York, 1943.

UNIVERSITY OF MARYLAND 